\title{
Validation of the Sexual Excitation/Sexual Inhibition Inventory \\ for Women and Men
}

Milhausen, R., Graham, C. A., Sanders, S. A., Yarber, W. L., \& Maitland, S. D. 


\begin{abstract}
The purpose of the current investigation was to develop a scale that would assess propensity for sexual arousal in response to a broad range of stimuli and sexual situations in both men and women. In Study 1, data from a nonclinical sample of 481 male and female students (graduate and undergraduate) were submitted to exploratory and confirmatory factor analyses in order to develop the Sexual Excitation Sexual Inhibition Scale for Women and Men (SESII-W/M), and gender differences on the subscales were tested. In Study 2, construct validity and test-retest reliability of the SESII-W/M were assessed with a second sample of undergraduate students. The resultant measurement tool was comprised of six subscales: Inhibitory Cognitions, Relationship Importance, Arousability, Partner Characteristics and Behaviors, Setting (Unusual or Unconcealed), and Dyadic Elements of the Sexual Interaction. The measure demonstrated good test-retest reliability and discriminant and convergent validity. The SESII-W/M will likely be a useful measure in investigations in which sexual inhibition and sexual excitation must be assessed identically for men and women.
\end{abstract}

Keywords: sexual arousal, sexual inhibition, sexual excitation, test validation 


\section{INTRODUCTION}

Most early sexual response theorists and researchers situated sexual arousal as an early stage in the sexual response cycle (Kaplan, 1979; Masters \& Johnson, 1966). In recent years, however, others have conceptualized arousal as the outcome of physiological, psychological, and behavioral processes (Janssen, Everaerd, Spiering, \& Janssen, 2000) and developed models to describe the processes that lead to sexual arousal in men and women (Bancroft, 1999; Bancroft \& Janssen, 2000; Graham, Sanders, Milhausen, \& McBride, 2004). For example, the dual control model of sexual response suggests that sexual arousal depends upon the relative activation of sexual excitation (SE) and sexual inhibition (SI), separate and independent systems (Bancroft, 1999; Bancroft \& Janssen, 2000). Individuals are assumed to vary in their capacity for both SE and SI. A certain level of SI is considered to be adaptive; however, individuals who have low levels of inhibition are likely to stay aroused in the presence of a potential threat or risk and less likely to take appropriate risk-reducing actions. Conversely, individuals with high levels of inhibition might be more vulnerable to sexual dysfunction. This model has been described more fully elsewhere (Bancroft, 1999; Bancroft \& Janssen, 2000).

To date, the majority of the research on the dual control model has been conducted using the Sexual Inhibition and Sexual Excitation Scales (SIS/SES), which were designed to assess the propensity for SE and SI in men (Janssen, Vorst, Finn, \& Bancroft, 2002a; 2002b). The SIS/SES has demonstrated strong reliability and validity. Factor analysis revealed ten first-order factors which loaded on three second-order factors: one excitatory (SES) and two inhibitory (SIS1 and SIS2). The ten-factor model fit only marginally better than the nested three-in-ten model, 
therefore, most subsequent research has focused on the three-factor structure (Bancroft, Graham, Janssen, \& Sanders, in press). SIS1 refers to inhibition due to the threat of performance failure (e.g., losing one's erection), whereas SIS2 refers to inhibition due to the threat of performance consequences (e.g., being caught having sex). The SIS/SES was adapted for women and used in a study of over 1,000 female college students (Carpenter, Janssen, Graham, Vorst, \& Wicherts, 2008). The data reasonably fit the factor structure obtained with the previous male samples (Janssen et al., 2002a). Women, as predicted, scored higher on SI and lower on SE compared to men; however, both women and men showed substantial variability in sexual inhibition and excitation scores, with close to normal distributions on all three subscales. Measures of reliability and validity were acceptable and similar to those found with male samples.

Although the SIS/SES demonstrated acceptable psychometric properties among women, we questioned whether the diversity of factors that could affect women's sexual arousal were adequately represented among the SIS/SES items. For example, relationship concerns and partner characteristics, which may be of particular importance to women (Ellison, 2001; The Working Group for a New View of Women's Sexual Problems, 2001), are under-represented on the SIS/SES. Secondly, it is also possible that different inhibitory mechanisms may be evident in women. Specifically, inhibitory patterns may be better developed in women (Bjorklund \& Kipp, 1996), women may be less variable in their tendency for inhibition than men (Bancroft, 1999), and inhibition may occur earlier on in a sexual interaction among women than among men (Graham et al., 2004; Tolman, 2001).

In response to these potential limitations of the SIS/SES measure for use with women, Graham, Sanders, and Milhausen (2006) developed a questionnaire grounded in women's experiences of factors affecting their sexual arousal, with items derived from a focus group study 
(Graham et al., 2004). Items reflected the wide range of factors which women indicated could impact their arousal at any given time, including: level of self-confidence; body image; mood or emotional state; physical condition (e.g., energy level, illness); partner characteristics (e.g., attractiveness, personality, intellect, and talent); relationship dynamics; elements of the sexual interaction (e.g., partner skill, setting); sexual or erotic stimuli; hormones; fertility, contraception and STD concerns; and alcohol and drug use. A total of 115 items were developed. The number of items was reduced using factor analysis and the measure was validated with a sample of 665 women. The resulting 36-item questionnaire, the Sexual Excitation/Sexual Inhibition Inventory for Women (SESII-W; Graham et al., 2006) consists of eight subscales. These were: Sexual Arousability (the tendency to become easily sexually aroused in a variety of situations); Partner Characteristics (the tendency for a partner's personality or behavior to impact on arousal); Sexual Power Dynamics (the tendency to be aroused by force or domination in a sexual situation); Smell (the tendency for olfactory cues to influence arousal); Setting (Unusual or Unconcealed) (the tendency for arousal to be enhanced by the possibility of being seen or heard while having sex); Concerns about Sexual Function (the propensity for worries about sexual functioning to influence arousal); Arousal Contingency (the potential for arousal to be easily inhibited or disrupted by situational factors); and Relationship Importance (reflecting the need for sex to occur within a specific type of relationship). The SESII-W measure demonstrated good discriminant and convergent validity and test-retest reliability (Graham et al., 2006).

Although one of our reasons for developing a new questionnaire designed to assess propensity for SI and SE in women was that the SIS/SES measure did not include items on themes that might be of particular relevance to women, we thought it likely that some of these themes might also be important for many men. A recent focus group study on factors or 
situations that facilitate or interfere with men's sexual arousal supports this (Janssen, McBride, Yarber, Hill, \& Butler, 2008). Following the focus group methodology used in the Graham et al. (2004) study, Janssen et al. asked 50, primarily heterosexual, men ranging in age from 18 to 70 years to describe factors that inhibit or enhance their sexual arousal. As found in women, men reported that feeling good about themselves and feeling desired by their partner facilitated their arousal. Also consistent with findings for women, the impact of negative mood on men's sexual arousal was variable. For some, stress and anger could facilitate, or at least not interfere with, their sexual arousal; for others, negative mood clearly reduced their sexual arousal. Partner characteristics, like attractiveness and intelligence, were important for many men. The majority of men reported that feeling emotionally "connected" to their partner enhanced their sexual arousal. For some men, particularly older men, emotional connection was integral to their arousal. This finding is supported by other research which suggests that relationship factors may be equally important to men's sexual functioning (McCabe, 1997). In a study focusing on the relationship between sexual desire and relationship functioning among community couples, Brezsnyak and Whisman (2004) found that higher levels of marital satisfaction predicted higher levels of sexual desire for husbands as well as for wives. Finally, many men, like women, noted that the context for the sexual interaction (e.g., having sex in a place where they could be seen or heard) and alcohol use could inhibit or enhance their arousal (Janssen et al., 2007).

Thus, it appears that many of the factors which women report influence their sexual arousal may have relevance for men. Although the SIS/SES questionnaire encompasses a number of these factors (e.g., elements of setting, partner arousal, partner attractiveness), others are not represented. The recent focus group study of men (Janssen et al., 2007) highlighted that partner characteristics and relationship dynamics may be particularly important influences on 
men's sexual arousal. A scale validated for use with both men and women that would assess propensity for sexual arousal in response to a broader range of stimuli and sexual situations could be useful for future research on gender differences and similarities. The purpose of the current investigation was to develop a scale that would assess factors that inhibit and enhance sexual arousal in men and women. In Study 1, exploratory and confirmatory factor analyses (EFA and CFA, respectively) were conducted to develop the Sexual Excitation Sexual Inhibition Scale for Women and Men (SESII-W/M), and gender differences on the subscales were tested. In Study 2, construct validity and test-retest reliability of the SESII-W/M were assessed.

Study 1

\section{METHOD}

\section{Participants}

Between November 2003 and January 2004, 4,000 email invitations to participate in the study were sent to undergraduate and graduate students at a large, Midwestern university. Participants were randomly selected by the Registrar's office from an electronic master list of students who met eligibility criteria. Eligibility criteria included being enrolled at the university and being 18 years of age or older. A total of 1390 individuals followed the email link to the online survey. Of these, 31 were not included in the analysis because they had technical problems with the survey $(\mathrm{N}=22)$ or they did not identify as male or female $(\mathrm{N}=9)$. Of the remaining 1359 (682 men, 677 women) a further 136 were excluded because they did not identify as heterosexual $(\mathrm{N}=111)$, or they were older than $36(\mathrm{~N}=25)$. Only students who identified as heterosexual were included in the analysis because previous research using the SESII-W suggests different response patterns based on sexual orientation (Graham, Sanders, Milhausen, \& McBride, 2003). The upper age limit of 36 was selected in order to eliminate 
participants who were more than three SDs from the mean in terms of age. In order to conduct exploratory and confirmatory factor analysis, no missing data in items used for modeling is permitted. Therefore, 742 participants were deleted from the analytic sample because they did not answer all of the SESII items (401 of these didn't answer any of the SESII items). The final sample was comprised of 481 participants (112 men and 369 women).

The mean age of participants was $21.62(\mathrm{SD}=3.53)$. The men were slightly but significantly older than the women $(22.51$ vs. $21.35, t(479)=3.07, p=.002)$. The participants were almost evenly split across the five school levels (freshman, sophomore, junior, senior, graduate/professional student), with slightly fewer participants at the freshman and sophomore level. The great majority of participants (90\%) identified as White. Just over one-half of the sample were in exclusive sexual relationships (55.2\%), 37.1\% reported not being in a sexual relationship, and $7.7 \%$ reported that they were in a non-exclusive relationship (see Table 1).

Fourteen percent had never engaged in penile-vaginal intercourse. There were no significant gender differences in terms of sexual experiences (e.g., age at first intercourse, number of sexual partners). The average age at first vaginal intercourse was $17.43(S D=2.41)$, the average number of lifetime partners was $6.00(S D=7.50)$, and the average number of vaginal intercourse partners over the past 12 months was $1.89(S D=1.89)$.

The response rate for the current study, 34.75\% (1390/4000) is higher than studies using comparable college samples and data collection methods (e.g., Kaplowitz, Hadlock, \& Levine, 2004, response rate of $25.4 \%$ with an email and reminder postcard; Sax, Gilmartin, \& Bryant, 2003, 21.5\%). However, the item response rate (surveys returned with all items complete; Sax, Gilmartin, \& Bryant) was much lower (12\% of total, and $34.6 \%$ of responders). It is difficult to 
compare this rate to other research because most researchers focus on total nonresponse as opposed to reporting the proportion of questionnaires completed with missing data.

Comparisons between those who answered all SESII items (the "completers," $N=481$ ) and those that completed only the demographic items (the "non-completers," $N=742$ ) revealed several significant differences. Although more men initially logged on to the website and completed the demographic items (682 men vs. 677 women), more women completed all of the SESII items (112 men vs. 369 women; $\left.\chi^{2}(1)=103.69, p<.001\right)$. Completers were more likely to identify as white $\left(92.5 \%\right.$ of the completers vs. $88.7 \%$ of the non-completers, $\chi^{2}(1)=4.85, p=$ $.03)$, and less likely to identify as Asian (3.5\% of the completers vs. $8.1 \%$ of the non-completers, $\left.\chi^{2}(1)=10.25, p=.001\right)$. Completers were more likely to report being freshman $(16.4 \%$ of completers vs. $10.9 \%$ of non-completers, $\left.\chi^{2}(1)=7.79, p=.005\right)$. There was no difference in terms of marital status and religiosity/spirituality.

\section{Measures}

\section{Demographic and Sexual History Questionnaire}

The questionnaire began with a number of items assessing demographic and sexual history (e.g., age, level of education, religion and religiosity, race, ethnicity, marital and relationship status, and sexual orientation). Sexual history items included age at first vaginal sex and lifetime number of vaginal sex partners. Participants were asked, "How old were you when you first engaged in penile-vaginal intercourse (penis inserted into the vagina)?" and "With how many different (male or female) partners have you engaged in sexual intercourse (penile-vaginal intercourse) in your lifetime?"

Sexual Excitation/Sexual Inhibition Inventory (SESII) 
The original SESII questionnaire, comprised of 115 items which refer to stimulus situations that could affect sexual inhibition and sexual excitation or to general statements about sexual arousal and inhibition, was the basis for the current investigation. The items were related to the eight categories identified in the previous focus group study (Graham et al., 2004): self (e.g., mood, body image, general health, concern regarding reputation); partner (e.g., physical appearance, personality); relationship dynamics/interaction (e.g., relationship quality); elements of the sexual interaction (e.g., timing, communication); setting (e.g., romantic, novel); sexual or erotic stimuli (e.g., fantasy, visual images); sexual and reproductive health (e.g., contraception, STDs); and alcohol/drug use. Two items were added to create versions of questions that would be appropriate for men. Specifically, the item "Women's bodies can really excite me sexually" was added to parallel "Men's bodies can really excite me sexually" and "I can become more easily aroused early in the morning" was added to parallel "I can become more easily aroused during certain times of my menstrual cycle." Thus, the total number of questionnaire items was 117.

The instructions to the questionnaire included the following: "Sometimes you may read a statement that is not applicable to you or a situation may have occurred in the past but is not likely to occur now. In such cases, please indicate how you think you would respond, if you were in that situation." Participants were asked to respond to the items on a 4-point Likert-type rating scale, from 1 (strongly disagree) to 4 (strongly agree).

\section{Procedure}

The Institutional Review Board for the Protection of Human Subjects approved all procedures. Between November 2003 and January 2004, each student selected in the random sample received an email from the principal investigator inviting him/her to participate in the study. The email included a link to a secure Web site. When an individual accessed the site, 
he/she read the Study Information Sheet and decided whether or not to participate in the study. By advancing to the next page, the student consented to participate and began answering demographic questions and the SESII items. To ensure respondent anonymity, no electronic individual user tracking data (for example, Internet Protocol [IP] addresses) were collected. A reminder email, including the Web address, was sent to the entire sample a week following the initial invitation.

The questionnaire was administered using a Web-based format. This method has many benefits, including cost-effectiveness, increased data accuracy, and increased response rates (Mustanski, 2001; Pealer, Weiler, Pigg, Miller, \& Dorman, 2001). Web-based questionnaires may elicit more honest responding, particularly regarding sensitive topics like sexual behaviors. Participants can complete the questionnaires, in most cases, with added convenience and privacy (Baer, Saroiu, \& Koutsky, 2002).

\section{Data Analysis}

In the first stage of data analysis, exploratory factor analysis (EFA) was used to determine the factor structure. Men's and women's data were analyzed together to maximize variability of responses and develop a factor structure that was inclusive of both genders. The data were analyzed using maximum likelihood factor analysis with promax rotation. Maximum likelihood factor analysis was selected because of the theoretical nature of the investigation. Oblique rotation was considered to be appropriate because the items were developed based on a theoretical model of independent sexual excitation and sexual inhibition factors, with potentially correlated subfactors within each. The data met assumptions for factor analysis such as those related to sample size, missing data, multicollinearity and singularity. 
Items were removed from the analysis in a systematic fashion following recommendations by Tabachnick and Fidell (2007). First, highly skewed items were removed (less than $10 \%$ in either of the two consecutive categories: strongly agree and agree or strongly disagree and disagree). Second, items with communalities lower than .30 were removed. In the subsequent analyses, items that had factor loadings lower than .40 , items that double-loaded, and items that made up single-item factors were removed.

Factor scale scores were created by calculating a mean of the items that loaded on each factor, with appropriate reverse coding of negatively loading items. For each participant, a score was calculated for each factor.

In the second stage of analysis, confirmatory factor analysis (CFA) was conducted. We employed CFA to test the structure of the SESII. Whereas EFA is exploratory in nature, CFA allows specification and tests of competing theoretical models. As such, CFA constitutes a more powerful statistical technique for assessing validity.

Models were tested using AMOS 7 (Arbuckle, 2006). Analyses were conducted on covariance matrices with results of the final models reported as standardized estimates for ease of interpretation. Factor scaling was accomplished by setting one factor loading to 1.0 for each factor. A test of a second-order factor was also conducted and factor scaling was achieved by fixing one indicator per factor to a value of 1.0 for all first- and second-order factors. The chisquare difference test ( $\Delta \chi^{2} ;$ Jöreskog \& Sörbom, 1987) was used to compare nested models and to evaluate whether model modifications provided significant improvement at each step. The critical value used for all comparisons was $p<.01$.

Model fit was evaluated by examining the following fit indexes: model $\chi^{2}$ and the ratio of $\chi^{2} / \mathrm{df}$ (Bollen, 1989); Goodness of Fit Index (GFI; Jöreskog \& Sörbom, 1987); Non-Normed 
Fit Index (NNFI; Bentler \& Bonnett, 1980); Comparative Fit Index (CFI; Bentler, 1990); and Root Mean Square Error of Approximation (RMSEA; Steiger, 1990). The model was determined to fit well if a consensus of measures met or exceeded generally accepted levels. It is widely accepted that model $\chi^{2}$ should be non-significant if the model fits well, although this measure is sensitive to large sample sizes. Therefore, we consulted GFI, NNFI, and CFI values and all these should exceed .9 to indicate acceptable fit. Additionally, RMSEA, which demonstrates the amount of error variance per degree of freedom in the model, should result in values smaller than .05 .

To address whether the factor solution was the same for men and women, we tested measurement equivalence/invariance of the best fitting model. First, the configural model assessed whether the factor structure was the same for men and women. Once the factor structure was established to be equivalent for men and women, three further hypotheses were tested: (a) equivalent factor loadings; (b) equivalent relationships between factors; and (c) the hypothesis of equivalent measurement residuals.

As the ability to detect gender differences in factors that inhibit and enhance arousal was a desirable outcome of scale development, analysis of variance was used to determine if men and women's scores on each of the factor subscales were significantly different. Cohen's $d$ effect sizes and confidence intervals were calculated for each of the significant comparisons. Effect sizes were calculated such that negative values of $d$ indicated women scoring higher on a factor and positive values of $d$ indicating men scoring higher.

\section{RESULTS}

\section{Exploratory Factor Analysis}


EFA resulted in a 6-factor solution that accounted for $38.62 \%$ of the variance. This was accepted as the final solution because it maximized the variance explained by the function and it represented a "clean solution" (i.e., no non-loading items, no double loading items, no single item factors). Generally, only variables with loadings greater than .32 should be interpreted (Comrey \& Lee, 1992). Items with loadings of .45 are considered fair, loadings of .55 are considered good, loadings of .63 are considered very good, and loadings of .71 and above are considered excellent. All items in the final factor structure met the .32 criterion. Nine of the 30 items were considered "fair," 12 of the items were considered "good," 8 were considered "very good,“ and 1 was considered "excellent” by this standard (Comrey \& Lee, 1992). All eigenvalues were greater than 1. Communalities for the 30 items ranged from .26 to .73, with 13 of the communalities higher than .40. Scree plots indicated that a 6-factor solution would sufficiently represent the SESII data. Finally, the factor solution was interpretable and theoretically meaningful. The factor scales were correlated at low levels (correlation coefficients ranged from -.01 to .40), with an average inter-factor correlation of .22.

Factor 1: Inhibitory Cognitions. Factor 1 consisted of eight items which predominantly pertain to cognitions or emotions that inhibit sexual arousal: worry about having an orgasm, worry about taking too long to become aroused, concern about being a good lover, and feeling shy or self-conscious during sex. Two items pertained to overall difficulty in becoming or staying aroused. Endorsement of items on this factor indicate that the individual's sexual arousal was negatively influenced by these concerns. Cronbach's alpha for this scale was .78.

Factor 2: Relationship Importance. Factor 2 consisted of five items which emphasize the importance of relationship security and quality to sexual arousal and the fear of being emotionally hurt or the possibility of being "used" as inhibitors of arousal. One item loading 
negatively pertained to sexual attraction as a more important prerequisite for sexual arousal than relationship status and was reverse coded to calculate the factor score. High scores on this factor indicate that sexual arousal is inhibited when it occurs in a relational context not characterized by trust and intimacy. Cronbach's alpha for this scale was .75.

Factor 3: Arousability. Factor 3 consisted of five items related to ease of arousability to various sexual stimuli. Items refer to arousal in response to thinking about someone who is very sexually attractive, talking about sex, and being physically close to a partner. Items about ease and frequency of arousability were also included. High scores on this factor scale suggest that the individual is more easily aroused. Cronbach's alpha for this scale was .72.

Factor 4: Partner Characteristics and Behaviors. Factor 4 consisted of five items related to partner characteristics and behaviors. Three items reflected partner characteristics (intelligence, talent, interpersonal skills) that influenced sexual arousal. Two items related to the impact of a partner performing specific behaviors on sexual arousal (doing chores, doing something "nice for me"). High scores on this factor scale suggest that positive partner characteristics positively influence sexual arousal. Cronbach's alpha for this scale was .71.

Factor 5: Setting (Unusual or Unconcealed). Factor 5 consisted of four items that relate to sexual arousal being enhanced by the sexual setting. Three of the four items refer to a risky setting in which others are nearby or the individual might get caught having sex. The fourth item refers to arousal in a novel setting. Two items were reverse coded for calculating the factor score. High scores on this factor imply that a person's arousal is enhanced when engaging in sexual behavior in an unusual or unconcealed setting. Cronbach's alpha for this scale was .76.

Factor 6: Dyadic Elements of the Sexual Interaction. Factor 6 consisted of three items that referred to partner variables during sexual activity that can inhibit arousal. Endorsing items 
loading on this factor indicates that negative partner dynamics during sexual interaction inhibit sexual arousal (e.g., partner insensitivity to sexual signals, lack of balance in giving and receiving during sex, and uncertainty about partner's feelings). Cronbach's alpha for this scale was .66.

\section{Confirmatory Factor Analysis}

We first examined whether a unidimensional (i.e., single factor) model would fit the SESII items. This model resulted in unacceptable fit: $\chi^{2}=2199.02, d f=405, \chi^{2} / \mathrm{df}=5.43, p<$ $.001, \mathrm{GFI}=.69, \mathrm{NNFI}=.42, \mathrm{CFI}=.46, \mathrm{RMSEA}=.10$.

Analysis of the six factors defined by 30 SESII items, including modeling correlations among all factors, resulted in good fit: $\chi^{2}=743.39, d f=390, \chi^{2} / \mathrm{df}=1.91, p<.001, \mathrm{GFI}=.91$, $\mathrm{NNFI}=.88, \mathrm{CFI}=.89, \mathrm{RMSEA}=.04$. All factor loadings were statistically significant and ranged from .45-.83, with an average standardized factor loading of .59. All factors were significantly intercorrelated except for: Inhibitory Cognitions and Partner Characteristics and Behaviors; Partner Characteristics and Behaviors and Setting; and Arousability and Dyadic Elements of the Sexual Interaction. Correlations between factors were in the expected directions and most were small to moderate, with an average inter-factor correlation of $r=.31$. Squared multiple correlations ranged from .23-.68, with an average SMC of .36 indicating that, on average, $36 \%$ of the variance in observed variables was accounted for by the latent factors.

Next, we examined a higher-order factor model to determine whether higher-order constructs of Sexual Inhibition and Sexual Excitation were plausible. A decrement in fit was

noted: $\chi^{2}=944.62, d f=401, \chi^{2} / \mathrm{df}=2.36, p<.001, \mathrm{GFI}=.89, \mathrm{NNFI}=.82, \mathrm{CFI}=.84, \mathrm{RMSEA}$ $=.05$. The loadings between the first-order Arousability factor and the second-order Sexual 
Excitation factor were marginally significant $(p=.013)$. Based on these results, we retained the model with six first-order factors as best fitting our data.

Finally, to address whether the factor solution was the same for men and women, we tested measurement equivalence/invariance of the best fitting model. The model fit the data equally well for men and women for the unconstrained, multigroup model and for two of the three subsequent constrained models. Results for the unconstrained model were: $\chi^{2}=1204.01$, $d f=780, \chi^{2} / \mathrm{df}=1.54, p<.001, \mathrm{GFI}=.86, \mathrm{NNFI}=.84, \mathrm{CFI}=.876 \mathrm{RMSEA}=.03$. The results for model constraining all factor loadings to be equivalent resulted in: $\chi^{2}=133.51, d f=804$, $\chi^{2} / \mathrm{df}=1.53, p<.001, \mathrm{GFI}=.86, \mathrm{NNFI}=.84, \mathrm{CFI}=.85, \mathrm{RMSEA}=.03$. Comparing the configural model to this model found the hypothesis of equivalent factor loadings was acceptable: $\Delta \chi^{2}=29.50, d f=24,(p=.202)$. Therefore, we tested the next level of stringency, constraining the factor variances and the relationships between factors to equivalence: $\Delta \chi^{2}=$ 36.66, $d f=21,(p=.018)$. This was also shown to be plausible. A test of whether the measurement residuals could be constrained to be equivalent was rejected. Therefore, sex invariance was accepted for the factor loadings and the variances and covariances between factors for this data, but not for the residuals. In summary, the six-factor solution worked well for both genders.

Creating the Sexual Excitation/Sexual Inhibition Inventory for Women and Men (SESIIW/M)

Responses to items $(N=30)$ loading on each of the six factors (Partner Characteristics and Behaviors, Setting (Unusual or Unconcealed), Dyadic Elements of the Sexual Interaction, Relationship Importance, Arousability, and Inhibitory Cognitions) were averaged to create factor scale scores. Table 2 presents the descriptive data for the factor scales. Each of the six factor 
scales fell within the normal range for skewness (between -1 and +1 ) and kurtosis (between -1 and +2) (Huck, 2000). Cronbach's alpha's for the factor scales ranged from .66 to .78; the mean alpha level was .73. Although published standards for acceptable alpha values vary, in general values between .70 and .90 are preferred for measures of sexual functioning. Scales with lower values may have inadequate internal consistency, whereas scales with higher values may be too narrow in scope (Daker-White, 2002). Five of the six factor scales met this criterion. These alpha levels were particularly appropriate given scores will be used to draw conclusions about groups (men and women) as opposed to individuals (Worthen, White, Fan, \& Sudweeks, 1999). Table 3 presents the items and factor loadings for each factor scale.

\section{Gender Differences on the SESII-W/M}

$T$-tests were used to determine if men's and women's scores on each of the six factors were significantly different (see Table 4). Women scored significantly higher than men on the Inhibitory Cognitions scales, the Relationship Importance scale, the Partner Characteristics and Behaviors Scale, and the Dyadic Elements of the Sexual Interaction scale. Men scored significantly higher than women on the Arousability scale and the Setting scale.

Hyde (2005) grouped Cohen's effect sizes in studies of gender differences in the following categories: trivial or close to zero $(d \leq 0.10)$, small $(0.11 \leq d \leq 0.35)$, moderate $(0.36 \leq$ $d \leq 0.65)$, large $(0.66 \leq d \leq 1.00)$, and very large $(d>1.00)$. In the current analysis, one comparison can be considered small (Dyadic Elements of the Sexual Interaction), three comparisons can be considered moderate (Arousability, Partner Characteristics and Behaviors, and Setting (Unusual or Unconcealed), and two can be considered very large (the Relationship Importance factor and the Inhibitory Cognitions factor) (see Table 4). 
In Study 1, the SESII-W/M was developed based on exploratory and confirmatory factor analysis. The objective for Study 2 was to assess the measure's construct validity and test-retest reliability.

\section{METHOD}

\section{Participants}

Participants were predominantly undergraduate students who were enrolled in distance education courses at a mid-sized Canadian university. Instructors of first- and second-year courses with enrolment of 50 or greater were contacted and asked to send email invitations to their students to complete the web-based questionnaire. Nineteen instructors were contacted and eight who taught a total of nine courses agreed to invite their students to participate. The enrolment for the combined 9 classes was 800 students.

A total of 175 students visited the website at Time 1 and completed the demographic questions. However, of those, 14 completed only the demographic questions and 12 did not answer at least $75 \%$ of items on the SESII-W/M scale. The validation analyses are therefore based on a sample of 149 participants (111 women and 38 men). Participants ranged in age from 17 to 38 , with the majority of participants (80\%) between the ages of 18 and 22 . The mean age of participants was $20.79(S D=3.29)$. Almost all identified as heterosexual $(92.6 \%)$. A minority identified as gay or lesbian (1.3\%), bisexual (4.7\%), or "other" (1.4\%). Almost onethird (29\%) were pursuing a Bachelor of Arts degree, over one-half were pursuing a Bachelor of Commerce degree, and the remainder were enrolled in various Bachelor of Science degrees programs. The majority of participants (82\%) identified as white and $14.3 \%$ identified as Asian. The students were distributed across every year in school (first through graduate) but the 
majority (58.4\%) were in first or second year. Most (43.6\%) were seriously dating one person or not dating anyone $(37.6 \%)$.

A total of 147 participants visited the survey website at Time 2. Eighteen did not complete any items past the demographic questions, and data from two individuals were excluded because they failed to complete at least $75 \%$ of all items on the SESII-W/M. Of the 127 remaining participants, 81 had completed the Time 1 questionnaire; thus, the test-retest sample was comprised of 81 participants (19 men, 62 women). The test-retest sample was not significantly different than the full sample in terms of sexual orientation, relationship status, year in school, ethnicity, or age. The proportion of men and women in each sample was not significantly different.

\section{Measures}

Identical measures used in the construct validation of the Sexual Excitation/Sexual Inhibition Inventory for Women (SESII-W) were used in the current study. A brief description of each measure and how it related to the factor scales in the Graham et al. (2006) study is included below. For more detailed information about these measures, see Graham et al. (2006). The Behavioral Inhibition/Behavioral Activation Scales (BIS/BAS; Carver \& White, 1994) This questionnaire measures two principal factors reflecting general behavioral inhibition and activation propensities: BIS (Behavioral Inhibition Scale, 7 items) and BAS (Behavioral Activation Scale). The Behavioral Activation Scale is comprised of three subscales: Reward Responsiveness (5 items), Drive (4 items), and Fun Seeking (4 items). Responses to the BIS/BAS items range from 1 (Very true for me) to 4 (Very false for me). The BIS/BAS was included to determine if the SESII-M/W measured distinctly sexual rather than general 
inhibition/activation propensities. The higher- and lower-order SESII-W factors showed small to moderate (.13 to .41$)$ correlations with the BIS/BAS scales, suggesting some shared variance between general inhibition and activation tendencies and sexual inhibition and excitation tendencies (Graham et al., 2006).

The Sexual Opinion Survey (SOS; Fisher, Byrne, White, \& Kelley, 1988)

The 21-item version of the SOS was used to measure erotophobia and erotophilia (the learned disposition to respond to sexual stimuli with negative-to-positive affect and evaluations). Response choices range from 1 (Strongly agree) to 7 (Strongly disagree). Scores on the SOS were significantly correlated with higher- and lower-order SESII-W factors; specifically, excitation factors were positively correlated ( $r$ 's between .24 and .53 ) with SOS and inhibition factors were negatively correlated ( $r$ 's between -.17 and -.41) (Graham et al., 2006).

Sexual Sensation Seeking Scale (SSS; Kalichman \& Rompa, 1995)

The 11-itemSSS measures propensity to pursue novel or risky sexual stimulation; response choices range from 1 (not at all like me) to 4 (very much like me). Scores on this measure have been positively correlated with sexual excitation proneness in men (Gaither \& Selbom, 2003). Among women, the SSS exhibited moderate correlations with the excitation factors of the SESII$\mathrm{W}(r$ 's between .31 and.58) and low to moderate negative correlations with the inhibition factors (r's between -.13 and -.39) (Graham et al., 2006).

Social Desirability Scale (SDSR; Hays, Hayashi, \& Stewart, 1989). The 5-item SDSR was used in the SESII-W validation study in order to determine to what degree items on the scale were influenced by social desirability. Responses to items on the SDSR range from 1 (Definitely true) to 5 (Definitely false). None of the correlation coefficients between the SESII-W factors and the SDSR scale exceeded .15, although some attained statistical significance (Graham et al., 2006). 


\section{Procedure}

Instructors forwarded their students the invitation email with a link to the study website. Students who clicked on the link were presented with the study information sheet and consent form, and those who agreed to participate were directed to the online SESII-W/M questionnaire. Approximately two weeks later, they were sent a second email invitation with a link to the identical questionnaire. All participants who visited the study website were given an opportunity to enter into a draw for a $\$ 50$ Visa gift card (completion of the questionnaire was not necessary).

\section{Data Analysis}

Validity was assessed by examining Pearson correlations between factor scores and scores on the other measures. Correlations between the SDSR and the factor scores were calculated to assess the effects of social desirability on responses to the SESII-MW. Internal consistency was assessed using Cronbach's alpha. Test-retest reliability was evaluated using Pearson's correlation coefficients.

\section{RESULTS}

\section{Test-Retest Reliability}

Mean factor scores and correlations for the first and second completions of the SESII$\mathrm{W} / \mathrm{M}$ are presented in Table 5. Correlations ranged from $r=.66$ to $r=.82$, with a mean correlation of .76. All correlations were significant at the $p<.005$ level.

\section{Construct Validity}

Most correlations between the SESII-W/M factors and the other measures were low to moderate and in the expected direction (see Table 6). Inhibitory Cognitions, an inhibitory factor, was correlated with BIS and negatively correlated with SOS and SSS. Similarly, Relationship Importance was negatively correlated with SOS and SSS. Arousability, Setting, and Partner 
Characteristics are excitatory factors, and each were positively correlated with the BAS total score and all BAS subscales. As expected, the highest correlations were between Arousability and Setting and the two sexuality measures, the SOS and the SSS. This is not surprising as these factors are comprised of items which refer to sexual stimuli or settings which enhance sexual arousal. One unexpected finding was the low $(r=.17)$ positive correlation between Dyadic Elements of the Sexual Interaction and BIS.

\section{DISCUSSION}

The current study presented the findings from two studies which validated the Sexual Excitation Sexual Inhibition Scale for Women and Men (SESII-W/M). In Study 1, exploratory factor analysis with a sample of 481 men and women identified a six-factor solution comprised of 30 items. Confirmatory factor analysis (CFA) indicated strong support for the six-factor model, and tests of sex invariance suggested the model fit equally well for men and women. The six factors identified were: Inhibitory Cognitions, Relationship Importance, Arousability, Partner Characteristics and Behaviors, Setting (Unusual or Unconcealed), and Dyadic Elements of the Sexual Interaction.

In Study 2, construct validity was assessed with a new sample of 149 men and women. The SESII-W/M largely correlated with other measures in the expected direction. The magnitude of these correlations suggests that our measure assesses different, although related, constructs. Specifically, the excitation factors Arousability, Setting, and Partner Characteristics and Behaviors showed low to moderate correlations with BAS (Behavioral Activation Scales; Carver $\&$ White, 1994), and moderate correlations with the SOS (Fisher et al., 1988) and SSS (Kalichman \& Rompa, 1995). Inhibitory Cognitions, an inhibition factor, was positively correlated with BIS, and negatively correlated with BAS, SOS, and SSS. This pattern of results 
was similar to those found with the SIS/SES (Janssen et al., 2002a), originally designed for men, and the SESII-W (Graham et al., 2006), originally designed for women. Test-retest reliability was assessed with a subsample of 79 participants who completed the questionnaire on two occasions (approximately 2 weeks apart); the average correlation of .76 indicates the measure has good test-retest reliability.

The factor structure in the current analysis was very similar to the SESII-W validation structure (Graham et al., 2006), although the samples were very different (females and males in the current sample vs. only females in the Graham et al. (2006) study; age range from 18 to 36 in the current sample vs. age range from 18 to 81 in the Graham et al. (2006) study). Nineteen of the 30 items in the SESII-W/M factor solution were also found in the SESII-W. In fact, the first five factors were highly similar in both solutions. Notable parallels include: the Setting factor (containing the identical items in both models), the Relationship Importance factor (sharing 4 of 5 items), the Inhibitory Cognitions factor (SESII-W/M) and the Concerns about Sexual Function Factor (SESII-W) (sharing 5 of 8 items), and the Arousability and Partner Characteristics and Behaviors factors (both sharing 3 of 5 items). Additionally, the factor solutions accounted for comparable amounts of variance. The consistency of findings across a sample of women only and the current sample provides added support for the model and suggests that these factors tap important aspects of sexual response for men as well as women.

Factor score distributions were normal, supporting the assumption that there is individual variability in propensity for sexual inhibition and sexual excitation. Women were more likely than men to report that positive partner characteristics enhanced their arousal. Women were also more likely to report that a lack of intimacy, generally, and specifically during the sexual encounter, could inhibit their sexual arousal. Also, women were more likely to endorse items 
related to inhibitory cognitions-thoughts, worries and concerns which they felt during the sexual encounter which decreased their sexual arousal. Men were more likely than women to indicate that a variety of sexual stimuli and settings could enhance their arousal.

The gender differences suggested by this analysis fit well with previous research on gender differences in sexual arousal and response. The largest and most striking gender difference occurred on the Relationship Importance factor. Women scored substantially higher on the factor than did their male counterparts. There is strong theoretical support, and some empirical support, for the supposition that women's sexual arousal is closely linked to their satisfaction with their partner and their relationship (Basson, 2000; 2001; Byers, 2001; The Working Group for a New View of Sexual Women's Sexual Problems, 2001). McCabe (1997) evaluated the differences in levels of intimacy between sexually functional and dysfunctional males and females and found women experienced sexual dysfunction even if only a few intimacy-related domains were lacking. For men, most aspects of intimacy had to be impaired before dysfunction occurred. This suggests a stronger relationship may exist between relationship quality and sexual functioning for women.

Also notable was the fact that women scored significantly higher than men on the factor Inhibitory Cognitions. Items from the Inhibitory Cognitions scale reflect concerns about sexual functioning, arousal contingency and performance anxiety. Inhibitory Cognitions, as conceptualized in the current study, is similar to SIS1 (Inhibition due to Threat of Performance Failure) from the SIS/SES (Janssen et al., 2002a). Janssen et al. originally conceptualized SIS1 as similar to "performance anxiety" but have alternatively considered SIS1 to measure inhibitory tone. Inhibitory tone can be described as the level of inhibition that the system is set at when not actively responding to a sexual stimulus or an external sexual threat (Bancroft \& Janssen, 2000). 
Analysis on a college sample of men and women revealed women had higher SIS1 scores than men (Carpenter et al., 2008). It may be that women have more developed inhibitory mechanisms than men (Bjorkland \& Kipp, 1996), for example, because of the risks associated with unplanned or unwanted pregnancy (Bancroft et al., in press).

Women also scored significantly higher on the Dyadic Elements of the Sexual Interaction factor. This new factor was not identified on the SESII-W; however, it is highly similar to the second SIS1 subscale, which describes inhibitions due to concerns during sexual interaction with a partner (Janssen et al., 2002a). The SIS1 subscale includes items referring to low partner arousal and concerns about pleasing one's partner. Hartman (1985) reported that sexually functional men experience an increase in sexual pleasure in response to signals of increasing pleasure from the partner. Ellison (2001) reported that one-quarter of the most important problems and concerns listed by women in a large convenience sample related to the partner's physical responsiveness or the woman's own physical responsiveness. A woman was more likely to consider a concern "problematic" if her partner had difficulty getting aroused or seemed distracted during sex. Further, women of all ages mentioned having partners who were not interested in performing oral or manual sex as often as they would have liked, perhaps indicating an imbalance of giving and receiving pleasure. Women's concerns regarding partner variables during sexual activity may have a more salient influence on their sexual arousal than men's.

Finally, women's scores on the Partner Characteristics and Behaviors scale were significantly higher than their male counterparts. Specifically, women reported more arousal in response to a partner displaying intelligence, talent, or social skills than did men. Evolutionary theorists suggest that women are the sexually selective sex, carefully choosing partners who appear to be healthy and attractive (as these are indicators of genetic quality) or who would be 
likely to invest in the relationship and offspring (Buss \& Schmidt, 1993). Thus, it would make sense that women's arousal would be influenced to a greater degree than men's when a partner displays desirable traits.

Men scored significantly higher on the Arousability factor. Research on masturbation (Gerressu, Mercer, Graham, Wellings, \& Johnson, 2008), sexual fantasy (Leitenberg \& Henning, 1995), frequency of thinking about sex, and desired number of sex partners supports this gender difference (Baumeister, Catanese, \& Vohs, 2001). The Arousability factor has elements in common with the Excitation subscales (SES) from the SIS/SES measure (Janssen et al., 2002a), particularly the first subscale (social interactions) and third subscale (thinking and fantasizing about sex). In a sample of college men and women, men had higher overall SES scores than did women (Carpenter et al., 2008). Another significant gender difference was found with regard to the Setting (Unusual or Unconcealed) factor, which contains items that refer to engaging in sexual activity in an unusual location or one where others might see or hear. Men scored higher on this factor than did women, indicating that their arousal was more often enhanced by sexual behavior in an unusual or unconcealed setting. In one study of men's and women's sexual fantasies (Davidson, 1985), several of the most common fantasies for women included sex in a room other than the bedroom or sex on a carpeted floor. The men fantasized about more exotic and exposed locales, such as sex on a beach. It appears then, that in some men and women, adventurous sex (to some degree) can facilitate sexual arousal; however, men's arousal is more likely to be enhanced in settings that are particularly novel or risky (with regard to potential discovery).

That men and women differed significantly on each of the factors related to sexual excitation or inhibition suggests that, although men and women may be more similar than 
different in a variety of arenas, the genders remain quite different on a number of domains related to sexuality (Hyde, 2005). It is interesting that this sample of men and women would demonstrate such large gender differences in sexual excitation and inhibition tendencies, given the striking similarity in terms of their reported sexual experiences. The within gender variability also appears to be predictive of sexual attitudes and behaviors.

These studies provide support for the use of the SESII-W/M as a measure of propensity for excitation and inhibition in response to various sexual stimuli and situations. Confirmatory factor analysis indicated the six-factor model was a good fit for the data, and that the model worked equally well for both genders. That the six-factor model is similar to the eight-factor model described in Graham et al. (2006), despite very different samples, indicates that the factors presented in both models, Inhibitory Cognitions, Relationship Importance, Arousability, Partner Characteristics and Behaviors, and Setting, may be meaningful across men and women at various life stages.

Findings of the study were subject to several limitations. First, a significant limitation was the use of a student sample, and the lack of racial and ethnic diversity. More research is needed to understand sexual arousal processes in underrepresented groups, and among middleaged and older adults. Although there are numerous parallels between these study findings and those of Graham et al. (2006) gathered with a sample of women ranging in age from _ _ , it is likely that factors which impact arousal change across the lifespan and in different relationship contexts. Additionally, although men and women were equally likely to follow the email link to the survey website, women were far more likely to complete all SESII items, leading to a gender imbalance. Research on gender differences in participation in online studies is mixed, some suggesting men are more likely to complete questionnaires while others indicating women are 
more frequent responders (Kaplowitz, Hadlock, \& Levine, 2004; Sax, Gilmartin, \& Bryant, 2003). We did not anticipate the lower completion rate for men and thus did not oversample men. Nonetheless, the overall response rate for this study is consistent with other online surveys, particularly those without personalized invitations, introductory postcards, or multiple electronic reminders (Kaplowitz et al., 2004; Sax et al., 2003). Future research internet research using the SESII-W/M would be strengthened by incorporating some of these strategies to increase responding and generate a more balanced sample in terms of gender. It is noteworthy that the analytic sample was comprised of 481 individuals, a "very good" sample size for factor analysis, according to Comrey and Lee (1992). Further tests of sex invariance with a larger sample of men would strengthen support for use of this measure with both genders.

Conducting online research has benefits and limitations. The ease of questionnaire completion for participants and the reduced measurement error are strengths of the current methodology (Baer et al., 2002). Nevertheless, important limitations need to be considered. Participants could have logged on and completed the questionnaire more than once, or forwarded the questionnaire link to others. Careful data cleaning was conducted, e.g., cases with unusual response patterns were deleted; however, it is impossible to completely correct for these issues. However, Web-based questionnaires are especially appropriate for use in college environments, where access to the Internet is becoming increasingly commonplace (Pealer et al., 2001). Further, web-based research may be uniquely appropriate for investigations focusing on sexuality. Participants completing online questionnaires have been found to skip fewer sensitive questions than those completing traditional pencil and paper surveys, perhaps because of greater perceived anonymity (Pealer et al., 2001). 
In recent years, a body of research based on the Dual Control Model has emerged, including studies using the SIS/SES and studies using the SESII-W. Research has linked propensity for sexual inhibition and sexual excitation to sexual risk-taking (Bancroft et al., 2004), the experience of sexual problems (Bancroft et al., 2005; Sanders et al., 2008), sexual orientation (Bancroft, Carnes, Janssen, \& Long, 2005; Sanders at el., 2008), and mood (Bancroft, Janssen, Strong, Vukadinovic, \& Long, 2003; Lykins, Janssen, \& Graham, 2006). The SESIIW/M includes a broad range of factors which can inhibit or enhance sexual arousal, factors which other research has shown to be relevant for both women and men (Graham et al., 2004; Janssen et al., 2008). Further, the measure has demonstrated structural and construct validity as well as test-retest reliability among both genders. Preliminary evidence suggests that the SESIIW/M will likely be a useful measure in investigations in which propensity for sexual inhibition and excitation must be assessed identically for men and women. Therefore, it could easily be incorporated into dyadic research in which the relative propensities for sexual arousal and inhibition would be of interest. Can it be used in clinical populations? 


\section{References}

Arbuckle, J. L. (2006). Amos 7.0 [Computer software]. Chicago, IL: Smallwaters.

Baer, A., Saroiu, S., \& Koutsky, L. A. (2002). Obtaining sensitive data through the Web: An example of design and methods. Epidemiology, 13, 640-645.

Bancroft, J. (1999). Central inhibition of sexual response in the male: A theoretical perspective. Neuroscience and Biobehavioral Reviews, 23, 763-784.

Bancroft, J., Carnes, J., Janssen, E., \& Long, J. S. (2005). Erectile and ejaculatory problems in gay and heterosexual men. Archives of Sexual Behavior, 34, 285-297.

Bancroft, J., Graham, C. A., Janssen, E., \& Sanders, S. A. (in press). The dual control model: Current status and future directions. Annual Review of Sex Research.

Bancroft, J., Herbenick, D., Barnes, T., Hallam-Jones, R., Wylie, K., Janssen, E., et al. (2005). The relevance of the dual control model to male sexual dysfunction: The Kinsey Institute/BASRT Collaborative Project. Sexual and Relationship Therapy, 20, 13-30.

Bancroft, J., \& Janssen, E. (2000). The dual control model of male sexual response: A theoretical approach to centrally mediated erectile dysfunction. Neuroscience and Biobehavioral Reviews, 24, 571-579.

Bancroft, J., Janssen, E., Carnes, L., Goodrich, D., Strong, D., \& Long, J. S. (2004). Sexual activity and risk taking in young heterosexual men: The relevance of sexual arousability, mood, and sensation seeking. Journal of Sex Research, 41, 181-192.

Bancroft, J., Janssen, E., Carnes, L., Strong, D. A., Goodrich, D., \& Long, J. S. (2004). Sexual activity and risk taking in young heterosexual men: The relevance of personality factors. 
Journal of Sex Research, 41, 181-192.

Bancroft, J., Janssen, E., Strong, D., Vukadinovic, Z., \& Long, J. S. (2003). The relation between mood and sexuality in heterosexual men. Archives of Sexual Behavior, 32, 217-230.

Basson, R. (2000). The female sexual response: A different model. Journal of Sex and Marital Therapy, 26, 51-65.

Basson, R. (2001). Human sex-response cycles. Journal of Sex and Marital Therapy, 27, 33-43.

Baumeister, R. F., Catanese, K. R., \& Vohs, K. D. (2001). Is there a gender difference in the strength of sex drive? Theoretical views, conceptual distinctions, and a review of relevant evidence. Personality and Social Psychology Review, 5, 2, 242-273.

Bentler, P. M. (1990). Fit indices, LaGrange multipliers, constraint changes, and incomplete data in structural models. Multivariate Behavioral Research, 25, 163-172.

Bentler, P. M., \& Bonnett, D. G. (1980). Significance tests and goodness-of-fit in the analysis of covariance structures. Psychological Bulletin, 88, 588-600.

Bjorklund, D. F., \& Kipp, K. (1996). Parental investment theory and gender differences in the evolution of inhibition mechanisms. Psychological Bulletin, 120, 163-188.

Bollen, K. A. (1989). Structural equations with latent variables. New York: John Wiley and Sons.

Brezsnyak, M., \& Whisman, M. A. (2004). Sexual desire and relationship functioning: The effects of marital satisfaction and power. Journal of Sex and Marital Therapy, 30, 199217.

Buss, D. M., \& Schmitt, D. P. (1993). Sexual strategies theory: An evolutionary perspective on human mating. Psychological Review, 100, 204-232. 
Byers, E. S. (2001). Evidence for the importance of relationship satisfaction for women's sexual functioning. Women and Therapy, 24, 23-26.

Carpenter, D. L., Janssen, E., Graham, C. A., Vorst, H., \& Wicherts, J. (2008). Women's scores on the Sexual Inhibition/Sexual Excitation Scales (SIS/SES): Gender similarities and differences. Journal of Sex Research, 45, 36-48.

Carver, C. S., \& White, T. L. (1994). Behavioral inhibition, behavioral activation, and affective responses to impending reward and punishment: The BIS/BAS Scales. Journal of Personality and Social Psychology, 67, 319-333.

Comrey, A. L., \& Lee, H. B. (1992). A first course in factor analysis $\left(2^{\text {nd }}\right.$ ed.). Hillsdale, NJ: Lawrence Erlbaum Associates.

Daker-White, G. (2002). Reliable and valid self-report outcome measures in sexual (dys)function: A systematic review. Archives of Sexual Behavior, 31, 197-209.

Davidson, J. K. (1985). The utilization of sexual fantasies by sexually experienced university students. Journal of American College Health, 34, 24-32.

Ellison, C. R. (2001). A research inquiry into some American women's sexual concerns and problems. Women and Therapy, 24, 147-159.

Fisher, W., Byrne, D., White, L. A., \& Kelley, K. (1988). Erotophobia-erotophilia as a dimension of personality. Journal of Sex Research, 25, 123-151.

Gaither, G. A., \& Sellbom, M. (2003). The Sexual Sensation Seeking Scale: Reliability and validity within a heterosexual college student sample. Journal of Personality Assessment, 81, 157-167. 
Gerressu, M., Mercer, C. H., Graham, C. A., Wellings, K., \& Johnson, A. M. (2008). British national probability data on masturbation prevalence and associated factors. Archives of Sexual Behavior, 37, 266-278.

Graham, C. A., Sanders, S., Milhausen, R. R., \& McBride, K. (November, 2003). Factors related to inhibition and excitation of sexual arousal in women. Paper presented at the meeting of the Society for the Scientific Study of Sexuality, Montreal, Quebec.

Graham, C. A., Sanders, S. A., Milhausen, R. R., \& McBride, K. R. (2004). Turning on and turning off: A focus group study of the factors that affect women's sexual arousal. Archives of Sexual Behavior, 33, 527-538.

Graham, C. A., Sanders, S. A., \& Milhausen, R. R. (2006). The Sexual Excitation and Sexual Inhibition Inventory for Women: Psychometric properties. Archives of Sexual Behavior, 35, 397-410.

Hartman, L. M. (1985). Attentional focus, sexual responding, and metacognitions. Journal of Sex Research, 21, 211-217.

Hays, R. D., Hayashi, T., \& Stewart, A. L. (1989). A five-item measure of socially desirable response set. Educational and Psychological Measurement, 49, 629-636.

Huck, S. W. (2000). Reading statistics and research ( $3^{\text {rd }}$ ed.). Boston; Allyn \& Bacon. Hyde, J. S-. (2005). The gender similarities hypothesis. American Psychologist, 60, 581-592. Janssen, E., Everaerd, W., Spiering, M., \& Janssen, J. (2000). Automatic processes and the appraisal of sexual stimuli: Toward an information processing model of sexual arousal. Journal of Sex Research, 37, 8-23. 
Janssen, E., McBride, K. R., Yarber, W., Hill, B. J., \& Butler, S. M. (2007). Factors that influence sexual arousal in men: A focus group study. Archives of Sexual Behavior, 37, 252-265.

Janssen, E., Vorst, H., Finn, P., \& Bancroft, J. (2002a). The Sexual Inhibition (SIS) and Sexual Excitation (SES) Scales: I. Measuring sexual inhibition and excitation proneness in men. Journal of Sex Research, 39, 114-126.

Janssen, E., Vorst, H., Finn, P., \& Bancroft, J. (2002b). The Sexual Inhibition (SIS) and Sexual Excitation (SES) Scales: II. Predicting psychophysiological response patterns. Journal of Sex Research, 39, 127-132.

Jöreskog, K. G., \& Sörbom, D. (1987). LISREL 7. A guide to the program and applications. Chicago: SPSS.

Kalichman, S. C., \& Rompa, D. (1995). Sexual Sensation Seeking and Sexual Compulsivity scales: Reliability, validity, and predicting HIV risk behavior. Journal of Personality Assessment, 65, 586-601.

Kaplan, H. S. (1979). Disorders of desire. New York: Brunner/Mazel.

Kaplowitz, M. D., Hadlock, T. D., \& Levine, R. (2004). A comparison of web and mail survey response rates. Public Opinion Quarterly, 68(1), 94-101.

Leitenberg, H., \& Henning, K. (1995). Sexual fantasy. Psychological Bulletin, 117, 469-496.

Lykins, A. D., Janssen, E., \& Graham, C. A. (2006). The relationship between negative mood and sexuality in heterosexual college women and men. Journal of Sex Research, 43, 136143.

Masters, W. H., \& Johnson, V. E. (1966). Human sexual response. London, England: Churchill. 
McCabe, M. P. (1997). Intimacy and quality of life among sexually dysfunctional males and females. Journal of Sex and Marital Therapy, 23, 276-290.

Mustanski, B. S. (2001). Getting wired: Exploiting the Internet for the collection of valid sexuality data. Journal of Sex Research, 38, 292-302.

Pealer, L. N., Weiler, R. M., Pigg, R. M., Miller, D., \& Dorman, S. M. (2001). The feasibility of a web-based surveillance system to collect health risk behavior data from college students. Health Education and Behavior, 28, 547-559.

Sanders, S. A., Graham, C. A., \& Milhausen, R. R. (2008). Predicting sexual problems in women: The relevance of sexual excitation and sexual inhibition. Archives of Sexual Behavior, 37, 241-251.

Sax, L., J., Gilmartin, S. K., \& Bryant, A. N. (2003). Assessing response rates and nonresponse bias in web and paper surveys. Research in Higher Education, 44(4), 409-432.

Steiger, J. H. (1990). Structural model evaluation and modification: An interval estimation approach. Multivariate Behavioral Research, 25, 173-180.

Tabachnick, B. G., \& Fidell, L. S. (2007). Using multivariate statistics (5th ed.). Boston: Allyn and Bacon.

The Working Group for A New View of Women's Sexual Problems. (2001). A new view of women's sexual problems. Women and Therapy, 24, 1-8.

Tolman, D. L. (2001). Female adolescent sexuality: An argument for the developmental perspective on the New View of Women's Sexual Problems. Women and Therapy, 24, 195-210.

Worthen, B. R., White, K. R., Fan, X., \& Sudweeks, R. R. (1999). Measurement and assessment in schools ( $2^{\text {nd }}$ ed.). New York: Longman. 
Table 1.

Study 1: Sample demographics

\begin{tabular}{|c|c|c|c|c|}
\hline \multirow[t]{2}{*}{ Demographic Variable } & $\begin{array}{l}\text { Women } \\
(\mathrm{N}=369)\end{array}$ & $\begin{array}{l}\text { Men } \\
(\mathrm{N}=112)\end{array}$ & $\chi^{2}$ & $p$ \\
\hline & $\mathrm{N}(\%)$ & $\mathrm{N}(\%)$ & & \\
\hline Hispanic or Latino/Latina & $15(4.1 \%)$ & $5(4.5 \%)$ & .032 & .86 \\
\hline \multicolumn{5}{|l|}{ Race/Ethnicity } \\
\hline White & $331(90.4 \%)$ & $101(91.8 \%)$ & 9.10 & .11 \\
\hline Asian & $6(1.6 \%)$ & $3(2.7 \%)$ & & \\
\hline Black/African American & $18(4.9 \%)$ & $0(0 \%)$ & & \\
\hline Hawaiian/OPI & $1(0.3 \%)$ & $1(.9 \%)$ & & \\
\hline Biracial or Multiracial & $10(2.7 \%)$ & $5(4.5 \%)$ & & \\
\hline \multicolumn{5}{|l|}{ Marital Status } \\
\hline Single/Never Married & $318(86.2 \%)$ & $93(83.0 \%)$ & & \\
\hline Living with Partner & $28(7.6 \%)$ & $8(7.1 \%)$ & & \\
\hline Married & $19(5.1 \%)$ & $10(8.9 \%)$ & & \\
\hline Separated/Divorced & $4(1.1 \%)$ & $1(.9 \%)$ & 2.19 & .53 \\
\hline \multicolumn{5}{|l|}{ Student Status } \\
\hline Freshman & $79(21.4 \%)$ & $0(0 \%)$ & & \\
\hline Sophomore & $68(18.4 \%)$ & $16(14.3 \%)$ & & \\
\hline Junior & $78(21.1 \%)$ & $27(24.1 \%)$ & & \\
\hline Senior & $77(20.9 \%)$ & $40(35.7 \%)$ & & \\
\hline Grad/Professional Student & $67(18.2 \%)$ & $29(25.9 \%)$ & 35.53 & .001 \\
\hline
\end{tabular}


Table 2.

Study 1: Descriptive results for the final six-factor solution $(N=481)$ landscape

\begin{tabular}{|c|c|c|c|c|c|c|c|}
\hline Factor & $\begin{array}{c}\% \text { of } \\
\text { Variance }\end{array}$ & $\mathrm{M}$ & SD & Range* & $\begin{array}{l}\text { Skew- } \\
\text { ness }\end{array}$ & Kurtosis & $\begin{array}{c}\text { Cronbach's } \\
\text { Alpha }\end{array}$ \\
\hline Inhibitory & 17.88 & 2.38 & .49 & $1.00-3.75$ & .025 & -.032 & .78 \\
\hline \multicolumn{8}{|l|}{ Cognitions } \\
\hline Relationship & 10.82 & 2.70 & .61 & $1.00-4.00$ & .009 & -.480 & .75 \\
\hline \multicolumn{8}{|l|}{ Importance } \\
\hline Arousability & 7.37 & 3.03 & .52 & $1.40-4.00$ & -.153 & -.336 & .72 \\
\hline Partner & 5.44 & 3.08 & .45 & $1.80-4.00$ & -.074 & -.286 & .71 \\
\hline \multicolumn{8}{|l|}{ Characteristics } \\
\hline \multicolumn{8}{|l|}{ And Behaviors } \\
\hline Setting & 4.79 & 2.63 & .62 & $1.25-4.00$ & -.093 & -.31 & .76 \\
\hline Dyadic & 4.15 & 3.01 & .54 & $1.00-4.00$ & -.271 & .198 & .66 \\
\hline \multicolumn{8}{|l|}{ Elements of the } \\
\hline Sexual & & & & & & & \\
\hline
\end{tabular}


Interaction

$* 1=$ Strongly disagree to $4=$ Strongly agree

Table 3 .

Study 1: Factor scales and factor loadings from Confirmatory Factor Analysis

\begin{tabular}{ll}
\hline Factor and Item & Factor
\end{tabular}

Loading

\section{Inhibitory Cognitions}

Sometimes I have so many worries that I am unable to get aroused.

If I feel that I am expected to respond sexually, I have difficulty getting aroused.

Sometimes I feel so "shy" or self-conscious during sex that I cannot become fully aroused.

If I think about whether I will have an orgasm, it is much harder for me to become aroused.

Unless things are "just right" it is difficult for me to become sexually aroused.

If I am worried about taking too long to become aroused, this can interfere with my arousal.

When I am having sex, I have to focus on my own sexual feelings in order to stay aroused.

If I am concerned about being a good lover, I am less likely to become 
aroused.

\section{Relationship Importance}

If I think that I am being used sexually it completely turns me off.

It would be hard for me to become sexually aroused with someone who is $\quad .62$ involved with another person.

If I am very sexually attracted to someone, I don't need to be in a $\quad-.60$ relationship with that person to become sexually aroused. (reverse coded) $\begin{array}{ll}\text { I really need to trust a partner to become fully aroused. } & .59\end{array}$ If I think that a partner might hurt me emotionally, I put the brakes on sexually.

\section{Arousability}

When I think about someone I find sexually attractive, I easily become sexually aroused.

I think about sex a lot when I am bored. becoming sexually aroused.

Just being physically close with a partner is enough to turn me on. 
Partner Characteristics and Behaviors

Seeing a partner doing something that shows his/her talent can make me very sexually aroused.

Someone doing something that shows he/she is intelligent turns me on. $\quad 63$

I find it arousing when a partner does something nice for me. $\quad .56$

If I see a partner interacting well with others, I am more easily sexually $\quad .54$ aroused.

If a partner surprises me by doing chores, it sparks my sexual interest.

Setting (Unusual or Unconcealed)

If it is possible someone might see or hear us having sex, it is more difficult $\quad .82$ for me to get aroused. (reverse coded)

I find it harder to get sexually aroused if other people are nearby. (reverse coded)

I get really turned on if I think I may get caught while having sex.

Having sex in a different setting than usual is a real turn on for me.

Dyadic Elements of the Sexual Interaction

If I am uncertain how my partner feels about me, it is harder for me to get 
aroused.

While having sex, it really decreases my arousal if my partner is not sensitive to the signals I am giving.

If interferes with my arousal if there is not a balance of giving and receiving $\quad .55$ pleasure during sex. 
Table 4.

Study 1: Gender differences on the SESII-W/M Factor Scales $(\mathrm{N}=481)$

\begin{tabular}{|c|c|c|c|c|c|c|}
\hline \multirow[t]{2}{*}{ Factor } & & \multirow[t]{2}{*}{ M } & \multirow[t]{2}{*}{ SD } & \multirow[t]{2}{*}{$t^{* *}$} & \multirow{2}{*}{$\begin{array}{l}\text { Effect } \\
\text { Size }\end{array}$} & \multirow{2}{*}{$\begin{array}{c}\text { Confidence } \\
\text { Interval }\end{array}$} \\
\hline & & & & & & \\
\hline \multirow[t]{2}{*}{ Inhibitory Cognitions } & Men & 2.02 & .44 & -9.97 & -1.04 & $-1.26--.0 .82$ \\
\hline & Women & 2.49 & .45 & & & \\
\hline \multirow[t]{2}{*}{ Relationship Importance } & Men & 2.22 & .54 & -10.34 & -1.12 & $-1.34--0.89$ \\
\hline & Women & 2.84 & .56 & & & \\
\hline \multirow[t]{2}{*}{ Arousability } & Men & 3.26 & .46 & 5.50 & .60 & $0.39-0.82$ \\
\hline & Women & 2.96 & .51 & & & \\
\hline Partner Characteristics and & Men & 2.89 & .43 & -5.25 & -0.56 & $-0.78--0.35$ \\
\hline Behaviors & Women & 3.14 & .44 & & & \\
\hline \multirow[t]{2}{*}{ Setting } & Men & 2.86 & .55 & 4.58 & 0.49 & $0.28-0.70$ \\
\hline & Women & 2.56 & .63 & & & \\
\hline Dyadic Elements of the & Men & 2.88 & .63 & -2.65 & -0.32 & $-0.53--0.11$ \\
\hline Sexual Interaction & Women & 3.05 & .50 & & & \\
\hline
\end{tabular}


Table 5

Study 2: Test-retest reliability $(\mathrm{N}=79)$

\begin{tabular}{|c|c|c|c|c|c|c|}
\hline \multirow[t]{2}{*}{ Factors } & \multicolumn{2}{|c|}{ Time 1} & \multicolumn{2}{|c|}{ Time 2} & \multirow[b]{2}{*}{$r$} & \multirow[b]{2}{*}{$p$} \\
\hline & M & SD & M & SD & & \\
\hline Inhibitory Cognitions & 2.31 & .50 & 2.44 & .55 & .80 & .001 \\
\hline Relationship Importance & 2.57 & .65 & 2.66 & .67 & .82 & .001 \\
\hline Arousability & 2.88 & .48 & 3.01 & .54 & .82 & .001 \\
\hline Setting & 2.59 & .54 & 2.60 & .63 & .75 & .001 \\
\hline Partner Characteristics and & 2.89 & .46 & 3.03 & .44 & .71 & .001 \\
\hline \multicolumn{7}{|l|}{ Behaviors } \\
\hline Dyadic Elements of the & 2.96 & .52 & 2.88 & .51 & .66 & .001 \\
\hline Sexual Interaction & & & & & & \\
\hline
\end{tabular}


Table 6.

Study 2: Correlations between the SESII-M/W and other measures $(\mathrm{N}=149)$

\begin{tabular}{|c|c|c|c|c|c|c|c|c|}
\hline Factors & $\begin{array}{c}\text { Social } \\
\text { Desirability }\end{array}$ & BIS & BAS & $\begin{array}{l}\text { BAS- } \\
\text { RR }\end{array}$ & $\begin{array}{c}\text { BAS- } \\
\text { D }\end{array}$ & $\begin{array}{c}\text { BAS- } \\
\text { FS }\end{array}$ & SOS & SSS \\
\hline Inhibitory Cognitions & .02 & $.26 * *$ & -.15 & -.04 & -.18 & -.11 & $-.19 *$ & $-30 * *$ \\
\hline Relationship & .13 & .13 & -.03 & -.01 & -.03 & -.03 & $-43 * *$ & $-48 * *$ \\
\hline \multicolumn{9}{|l|}{ Importance } \\
\hline Arousability & -.13 & .08 & $.28 * *$ & $.32 * *$ & $.20 *$ & $.17 *$ & $.48 * *$ & $.53 * *$ \\
\hline Setting & -.06 & -.03 & $.24 * *$ & .12 & $.22 * *$ & $.21 * *$ & $.44 * *$ & $.48 * *$ \\
\hline Partner Characteristics & -.01 & $.26 * *$ & $.26 * *$ & $.24 * *$ & $.21 * *$ & $.18 *$ & -.01 & .02 \\
\hline and Behaviors & & & & & & & & \\
\hline $\begin{array}{l}\text { Dyadic Elements of } \\
\text { the Sexual Interaction }\end{array}$ & .06 & $.17 *$ & -.05 & .10 & -.16 & -.02 & -.16 & -.17 \\
\hline$* \mathrm{p}<.05$ & & & & & & & & \\
\hline
\end{tabular}


Figure 1. Confirmatory Factor Analysis: Six-factor model

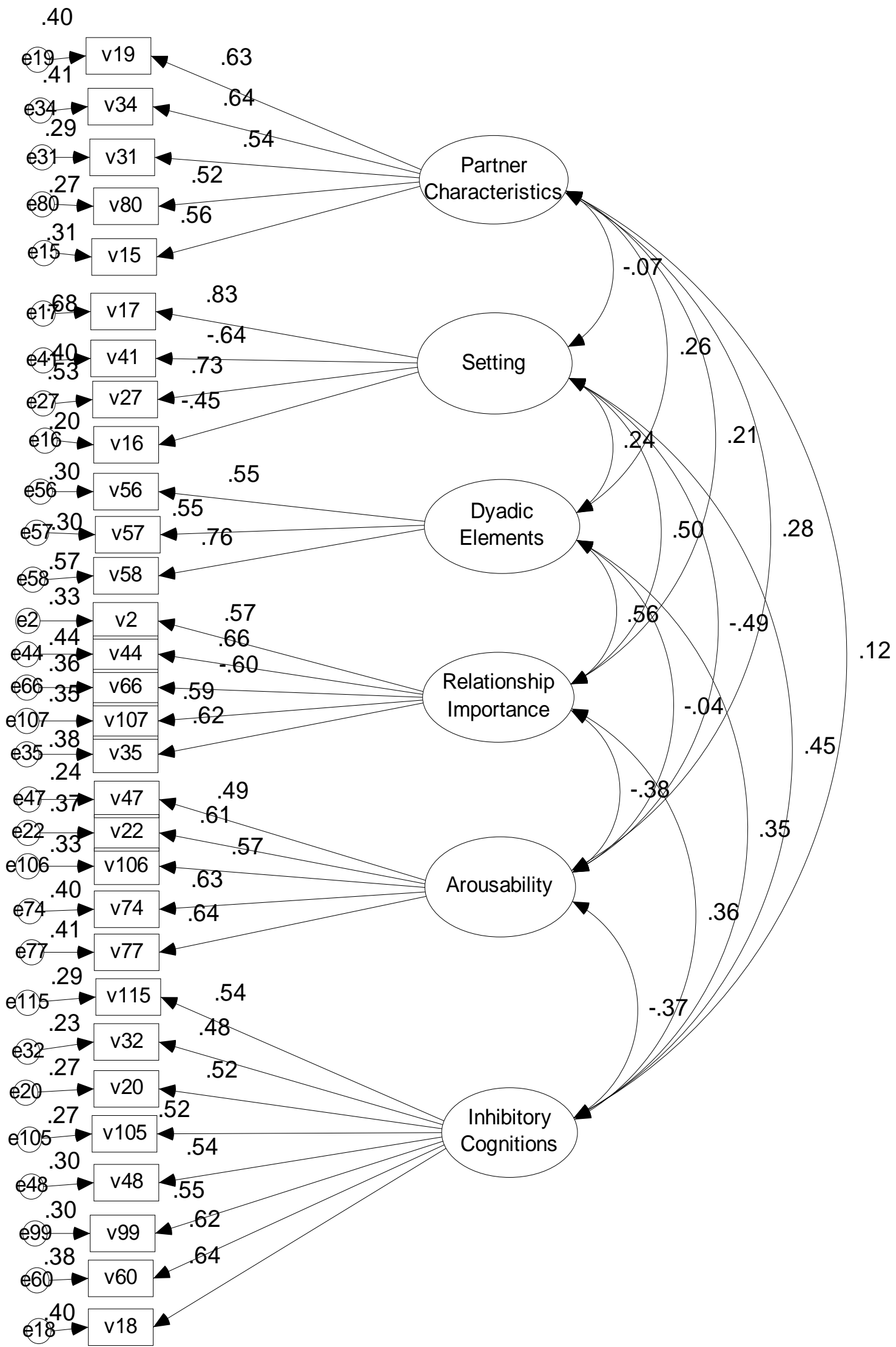

